(1)

CrossMark

\title{
Phenotype of asthmatics with increased airway S-nitrosoglutathione reductase activity
}

\author{
Nadzeya V. Marozkina ${ }^{1}$, Xin-Qun Wang ${ }^{2}$, Vitali Stsiapura ${ }^{3}$, Anne Fitzpatrick ${ }^{4}$, \\ Silvia Carraro ${ }^{5}$, Gregory A. Hawkins ${ }^{6}$, Eugene Bleecker ${ }^{6}$, Deborah Meyers $^{6}$, \\ Nizar Jarjour ${ }^{7}$, Sean B. Fain ${ }^{8}$, Sally Wenzel ${ }^{9}$, William Busse ${ }^{7}$, Mario Castro $^{10}$ \\ Reynold A. Panettieri Jr ${ }^{11}$, Wendy Moore ${ }^{6}$, Stephen J. Lewis ${ }^{1}$, Lisa A. Palmer ${ }^{12}$ \\ Talissa Altes $^{13}$, Eduard E. de Lange ${ }^{13}$, Serpil Erzurum ${ }^{14,15}$, W. Gerald Teague ${ }^{12}$ \\ and Benjamin Gaston ${ }^{1}$
}

Affiliations: ${ }^{1}$ Dept of Paediatrics, Rainbow Babies and Children's Hospital, Case Western Reserve University, Cleveland, $\mathrm{OH}$, USA. ${ }^{2}$ Dept of Public Health Sciences, University of Virginia, Charlottesville, VA, USA. ${ }^{3}$ Dept of Chemistry, University of Virginia, Charlottesville, VA, USA. 'Dept of Paediatrics, Emory University, Atlanta, GA, USA. ${ }^{5}$ Dept of Paediatrics, University of Padua, Padua, Italy. ${ }^{6}$ Dept of Medicine, Wake Forest University, Winston-Salem, NC, USA. ${ }^{7}$ Dept of Medicine, University of Wisconsin, Madison, WI, USA. ${ }^{8}$ Dept of Medical Physics, University of Wisconsin, Madison, WI, USA. ${ }^{9}$ University of Pittsburgh, Pittsburgh, PA, USA. ${ }^{10}$ Dept of Medicine, Washington University, St. Louis, MO, USA. ${ }^{11}$ Pulmonary, Allergy and Critical Care Division, University of Pennsylvania School of Medicine, Philadelphia, PA, USA. ${ }^{12}$ Dept of Paediatrics, University of Virginia, Charlottesville, VA, USA. ${ }^{13}$ Dept of Radiology and Medical Imaging. University of Virginia, Charlottesville, VA, USA. ${ }^{14}$ Dept of Pathobiology, Cleveland Clinic, Cleveland, OH, USA. ${ }^{15}$ Dept of Pulmonary, Allergy, and Critical Care Medicine, Cleveland Clinic, Cleveland, $\mathrm{OH}$, USA.

Correspondence: Benjamin Gaston, Rainbow Babies and Children's Hospital, Case Western Reserve University School of Medicine, Paediatric Pulmonology, BRB Room 727, 10900 Euclid Ave, Cleveland, OH 44106, USA. E-mail: benjamin.gastondacase.edu

ABSTRACT S-Nitrosoglutathione is an endogenous airway smooth muscle relaxant. Increased airway $S$-nitrosoglutathione breakdown occurs in some asthma patients. We asked whether patients with increased airway catabolism of this molecule had clinical features that distinguished them from other asthma patients.

We measured $S$-nitrosoglutathione reductase expression and activity in bronchoscopy samples taken from 66 subjects in the Severe Asthma Research Program. We also analysed phenotype and genotype data taken from the program as a whole.

Airway $S$-nitrosoglutathione reductase activity was increased in asthma patients $(p=0.032)$. However, only a subpopulation was affected and this subpopulation was not defined by a "severe asthma" diagnosis. Subjects with increased activity were younger, had higher IgE and an earlier onset of symptoms. Consistent with a link between $S$-nitrosoglutathione biochemistry and atopy: 1) interleukin 13 increased $S$-nitrosoglutathione reductase expression and 2) subjects with an $S$-nitrosoglutathione reductase single nucleotide polymorphism previously associated with asthma had higher IgE than those without this single nucleotide polymorphism. Expression was higher in airway epithelium than in smooth muscle and was increased in regions of the asthmatic lung with decreased airflow.

An early-onset, allergic phenotype characterises the asthma population with increased S-nitrosoglutathione reductase activity.

@ERSpublications

Asthma patients with increased $S$-nitrosoglutathione reductase activity have early-onset asthma + are highly allergic http://ow.ly/AuJ9z

This version of this article has been amended. The amendment is outlined in the erratum published in the June 2015 issue of the European Respiratory Journal [DOI: 10.1183/09031936.50042414].

Received: March 042014 | Accepted after revision: Aug 122014 | First published online: Oct 302014

Copyright @ERS 2015 


\section{Introduction}

$S$-Nitrosoglutathione (GSNO) is one of a class of endogenous sulphur nitric oxide bond-containing species (S-nitrosothiols) that serve as signalling molecules and airway smooth muscle relaxants [1-5]. Nitrosylating and denitrosylating enzymes regulate the formation and breakdown of $S$-nitrosothiols [4-8]. In the cellular environment, $S$-nitrosylated proteins are in transnitrosation equilibria with reduced glutathione (GSH) [4-8]. Thus, enzymatic denitrosylation of GSNO depletes S-nitrosylated proteins in a given cell or compartment. The most extensively studied denitrosylating enzyme in the lung is GSNO reductase, also known as alcohol dehydrogenase 5 (ADH5) $[4-6,9,10]$.

GSH, with its redox cycle partners, serves to maintain the reduced state of protein thiols, which can be achieved by scavenging oxidants or by the covalent reversible binding of GSH to protein thiols. The latter occurs under physiological conditions, is induced upon mild oxidative stress and is known as S-glutathionylation (PSSG) [11]. GSH can be removed from proteins by glutaredoxins (Grx), which restores the function of proteins targeted by PSSG [11]. It has been shown by KuIPERS et al. [12] that in the sputum of asthmatics there is increased Grx-1 and decreased protein S-glutathionylation.

Levels of GSNO and other $S$-nitrosothiols are also decreased in the airway lining fluid of many patients with mild asthma, and intratracheal levels are profoundly low in children with acute asthmatic respiratory failure $[10,13]$. The GSNO reductase-deficient mouse is protected from methacholine-induced bronchoconstriction [9] and endogenous $S$-nitrosothiols prevent tachyphylaxis to $\beta_{2}$-adrenergic agonists [3]. Further, inhaled GSNO decreases airway levels of interleukin (IL)-4, -5, -6 and -13, as well as other mediators associated with allergic airway inflammation [5]. Indeed, GSNO reductase gene single nucleotide polymorphisms (SNPs) are associated with increased asthma incidence in specific human asthma subpopulations [14-16]. Thus, it is suggested that GSNO replacement, and/or the inhibition of GSNO reductase, could attenuate asthma symptoms in humans [17].

However, of concern is that long-term treatment, aimed at substantially increasing airway levels of $S$-nitrosothiols in patients who already have normal levels, could, theoretically, predispose them to adverse consequences [18-20] or could, paradoxically, increase airway hyperresponsiveness at higher doses [5]. Furthermore, most patients with mild asthma have normal airway levels of epithelial GSNO reductase activity [10]. Therefore, not all asthma patients should be treated with $S$-nitrosothiol repletion or GSNO reductase inhibition. Here, our goal has been to identify the determinants of increased GSNO reductase activity and the phenotypic features of this subpopulation of asthma patients. With this information, personalised therapy might be envisioned for those who are most likely to respond.

Personalised asthma treatment is particularly important for severe asthma. Patient response to standard therapies, including leukotriene receptor antagonists and inhaled corticosteroids, is suboptimal in many patients with severe asthma $[21,22]$. Targeting the most probable responders is likely to decrease both the cost and the toxicity of the treatment. Furthermore, severe asthma patients are defined as those who are highly symptomatic despite these treatments; these patients may particularly benefit from a medical approach in which responsiveness is suspected based on a clinical phenotype, diagnostic test and targeted treatment. For example, specific recommendations on the use of sputum eosinophil count and exhaled nitric oxide to guide therapy, as well as treatment with anti-IgE antibody, methotrexate, macrolide antibiotics, antifungal agents and bronchial thermoplasty are provided in the International European Respiratory Society/American Thoracic Society (ATS) guidelines on definition, evaluation and treatment of severe asthma [23]. Here, we have begun to approach this type of paradigm by studying the phenotype of the asthma subpopulation with increased airway GSNO reductase activity.

\section{Methods}

Subjects

Non-smoking subjects with severe and nonsevere asthma, as well as healthy controls, who had enrolled in the Severe Asthma Research Program (SARP) underwent previously described characterisation procedures [21] using the ATS consensus pane definition of [22]. A subpopulation underwent hyperpolarised helium (HHe-3) magnetic resonance imaging (MRI) [24]. Not all subjects underwent all tests. All procedures were approved by the Institutional Review Board at the SARP centres, and participants provided informed consent.

Support statement: B. Gaston received funding for this study from: NIH Severe Asthma Research Program Grant \#5U10HL109250-02; NIH Program Project Grant \#5P01HL101871-02; and NIH R01 Grant \#5RO1-HL059337-12. R.A. Panettieri has received funding for this study from NIH R01 Grant \#HL097796 and NIEHS P30 ES013508. E.E. de Lang has received funding for this study from NIH RO1 HL66479.

Conflict of interest: Disclosures can be found alongside the online version of this article at erj.ersjournals.com 


\section{Bronchoscopy}

Adult subjects underwent flexible bronchoscopy with BAL and endobronchial biopsies of segmental carinae, using our published methods [25]. Children with severe asthma underwent bronchoscopy for clinical indications according to a shared sample protocol at Emory University (Atlanta, GA, USA) [26]. Biopsies were paraffin embedded and subjected to immunohistochemistry and haematoxylin/eosin staining. At Emory University, the lavage fluid was aspirated in initial and terminal phase fractions [26]. To identify well- and poorly-ventilated lung regions, three subjects scheduled for bronchoscopy underwent HHe-3 MRI; within $12 \mathrm{~h}$, biopsies were performed in airways with and without ventilatory defects.

Imaging

Subjects underwent HHe-3 MRI according to an established protocol [24, 27].

\section{Biochemical assays}

GSNO reductase was measured as NADH-dependent loss of GSNO over time using two separate methods with strong concordance: 1) mass spectrometry and 2) spectrophotometry [18, 28].

\section{Cell culture}

Primary human airway smooth muscle (HASM) cells, isolated in the laboratory of Reynold A. Jr Panettieri, were cultured as previously described [29]. Normal human bronchial epithelial cells (NHBE) (Lonza, Walkersville, MD, USA) were grown as monolayers, and primary human bronchial epithelial cells were grown at air-liquid interface as previously described [30]. Cystic fibrosis bronchial epithelial (CFBE41o-) and A549 cell lines were grown as previously described [19, 30].

\section{Immunoblot}

GSNO reductase AB (1:1000) (\#11051-1-AP; Proteintech Group, Chicago, IL, USA) and second HRP 1:10 000 was used to immunostain for HASM, and CFBE cells. Additionally, airway expression was confirmed using a separate polyclonal rabbit anti-GSNO reductase AB kindly donated by L. Que (Duke University, NC, USA) with goat anti-rabbit secondary AB (\#172-1019; Bio-Rad Inc., Hercules, CA, USA).

\section{Immunohistochemistry}

Tissue sections were immunoblotted with GSNO reductase AB (1:1000) (\#11051-1-AP; Proteintech Group) using secondary goat anti-rabbit $(1: 10$ 000) for $30 \mathrm{~min}$ at room temperature (\#11051-1-AP; Proteintech Group) and ABC reagent (Vector Laboratories, Burlingame, CA, USA) for 30 min at room temperature, as previously described [19], and scored from 0 (absent) to 4 (intense staining) by two observers who were blinded to the diagnosis.

\section{SNP genotyping and assessment}

The ADH5 genotypes were acquired from the Human1M-Duo DNA BeadChip (Illumina, San Diego, CA, USA). The Hardy-Weinberg equilibrium was tested for quality control.

\section{Statistical analysis}

By univariate analysis, we studied the relationship between GSNO reductase activity and risk factors. A t-test or nonparametric rank sum test was used for two-sample comparison of continuous variables, a Fisher exact test was used for two-sample comparison of categorical variables, and analysis of variance was used for categorical variables with more than two categories. $\mathrm{p}<0.05$ was considered statistically significant. The statistical analyses were carried out in SAS 9.2 (SAS Institute Inc., Cary, NC, USA).

\section{Results}

\section{Subjects}

We studied 52 adults (healthy controls $n=12$, nonsevere asthma $n=25$, and severe asthma $n=15$ ) and 14 children (healthy controls $n=2$, nonsevere asthma $n=6$, and severe asthma $n=6$ ) (table 1). These subjects were enrolled at five different sites participating in SARP. Through site-specific protocols, subsets of subjects underwent specific procedure (biopsy, bronchoalveolar lavage (BAL) and/or MRI). BALs were performed in the right middle lobe or lingula. Except where directed by MRI, biopsies were performed at a mainstem, third or fourth generation carina contralateral to the BAL site. Genetic analysis was additionally performed on 914 asthma subjects and 344 controls obtained from sites participating in SARP.

\section{GSNO reductase activity}

We measured GSNO reductase activity in 54 BAL cell lysates (patients with severe asthma $\mathrm{n}=16$, nonsevere asthma $n=25$, and controls $n=13$ ). Overall the mean values did not differ between the three groups; the p-value was nonsignificant (NS). There was a subpopulation of $44 \%$ of the asthma patients with 
TABLE 1 Characteristics of subjects who underwent bronchoscopy

\begin{tabular}{|c|c|c|c|c|}
\hline & Control & Nonsevere asthma & Severe asthma & ANOVA \\
\hline \multicolumn{5}{|l|}{ Adults SARP-wide } \\
\hline Subjects & 12 & 25 & 15 & \\
\hline Male & 7 & 8 & 6 & NS \\
\hline Female & 5 & 17 & 9 & \\
\hline Age years & $27 \pm 5.9$ & $32 \pm 11$ & $39 \pm 17$ & $p<0.02$ \\
\hline Race & & & & NS \\
\hline Caucasian & 10 & 20 & 9 & \\
\hline African-American & 1 & 3 & 3 & \\
\hline Asian & 1 & 0 & 0 & \\
\hline Other & 0 & 1 & 0 & \\
\hline Multiple & 0 & 1 & 3 & \\
\hline Body mass index $\mathrm{kg} \cdot \mathrm{m}^{-2}$ & $26 \pm 4.8$ & $27 \pm 5.6$ & $29 \pm 6.8$ & NS \\
\hline \multicolumn{5}{|l|}{ Pre-bronchodilator } \\
\hline $\mathrm{FEV}_{1} \%$ & $95 \pm 8.9$ & $90 \pm 14$ & $67 \pm 20$ & $p<0.0001$ \\
\hline FVC \% & $100 \pm 11$ & $96 \pm 13$ & $82 \pm 15$ & $p<0.0006$ \\
\hline $\mathrm{FEV}_{1} / \mathrm{FVC}$ & $0.79 \pm 0.05$ & $0.78 \pm 0.1$ & $0.67 \pm 0.09$ & $p<0.0006$ \\
\hline $\mathrm{PC}_{20 \mathrm{MC}} \mathrm{mg} \cdot \mathrm{mL}^{-1}$ & $41 \pm 16$ & $6.8 \pm 11.4$ & $2.8 \pm 6.0^{7}$ & $p<0.0001$ \\
\hline $\operatorname{lgE}$ blood IU.mL ${ }^{-1}$ & $32 \pm 36$ & $407 \pm 1147^{\#}$ & $230 \pm 290^{+}$ & NS \\
\hline On any inhaled steroid \% & 0 & 48 & 100 & \\
\hline Number of positive skin reactions & $1.2 \pm 1.9$ & $4.2 \pm 3.0^{\#}$ & $4.1 \pm 2.2^{\S}$ & $p<0.01$ \\
\hline \multicolumn{5}{|l|}{ Children SARP-wide } \\
\hline Subjects & 2 & 6 & 6 & NS \\
\hline Male & 0 & 4 & 3 & \\
\hline Female & 2 & 2 & 3 & \\
\hline Age years & $16 \pm 0.7$ & $11 \pm 3.2$ & $11 \pm 4.2$ & NS \\
\hline Race & & & & $p<0.004$ \\
\hline Caucasian & 2 & 5 & 1 & \\
\hline African-American & 0 & 1 & 5 & \\
\hline Body mass index $\mathrm{kg} \cdot \mathrm{m}^{-2}$ & $33 \pm 15$ & $22 \pm 10$ & $17 \pm 3.4$ & $p<0.05$ \\
\hline \multicolumn{5}{|l|}{ Pre-bronchodilator } \\
\hline $\mathrm{FEV}_{1} \%$ & $95 \pm 0.7$ & $100 \pm 15$ & $76 \pm 19$ & NS \\
\hline FVC $\%$ & $94 \pm 5.7$ & $106 \pm 17$ & $82 \pm 26$ & NS \\
\hline $\mathrm{FEV}_{1} / \mathrm{FVC}$ & $0.88 \pm 0.04$ & $0.87 \pm 0.09$ & $0.65 \pm 0.14$ & $p<0.009$ \\
\hline $\lg \mathrm{E}$ serum $\mathrm{kU} \cdot \mathrm{L}^{-1}$ & $67 \pm 78$ & $140 \pm 148$ & $1025 \pm 1081$ & NS \\
\hline On any inhaled steroid \% & 0 & 83 & 100 & \\
\hline
\end{tabular}

Data are presented as $\mathrm{n}$ or group mean \pm SD, unless otherwise stated. SARP: Severe Asthma Research Program; FEV1, forced expiratory volume in $1 \mathrm{~s}$; FVC: forced vital capacity; PC20: provocative concentration causing a $20 \%$ fall in FEV1; MC: methacholine; Ns: nonsignificant. ${ }^{\#}: n=24 ;{ }^{9}: n=9 ;{ }^{+}: n=14 ;{ }^{\S}: n=13$.

levels $>7.5 \mathrm{nmol} \cdot \mathrm{min}^{-1} \cdot \mu^{-1}$ protein (more in asthma than controls, $\mathrm{p}=0.032$ ) (fig. 1a). We performed immunoblot for GSNO reductase on a subpopulation of nine BAL samples (control $n=1$, severe $n=1$, and nonsevere $\mathrm{n}=7$ ). There was a weak concordance between measures of GSNO reductase activity in airway versus alveolar lavage samples $\left(n=11, r^{2}=0.61\right)$ (fig. $1 \mathrm{~b}$ and $\mathrm{c}$ ) and between BAL GSNO reductase expression and activity $\left(\mathrm{r}^{2}=0.54\right)$ (fig. $1 \mathrm{c}$ ), which were consistent with a previous report [10]. Activity did not vary with BAL cell count, differential or other parameters.

\section{Tissue GSNO reductase expression}

Next, we studied GSNO reductase tissue expression in random endobronchial biopsies (i.e. not directed by HHe3 imaging; $n=18$, which included: severe asthma $n=6$, nonsevere asthma $n=4$ and controls $n=8)$. There was less GSNO reductase expression in smooth muscle cells than in epithelial cells $(\mathrm{p}<0.05)$ (fig. 2). However, GSNO reductase immunoreactivity did not differ between asthma and control patients in epithelial, smooth muscle or inflammatory cells. Immunoreactivity localised primarily to the apical regions of airway epithelial cells of healthy lungs. This was not the case in epithelium from asthmatic subjects, where immunoreactivity localised to basal cells (fig. 2). Consistent with these data, HASM cells expressed less GSNO reductase, relative to protein load control, than NHBE cells in cultured monolayers, NHBE cells grown as pseudostratified epithelium at the air-fluid interface and the established airway cell lines CFBE (fig. 3a). Of note, interleukin (IL) 13 increased epithelial GSNO reductase expression in severe 

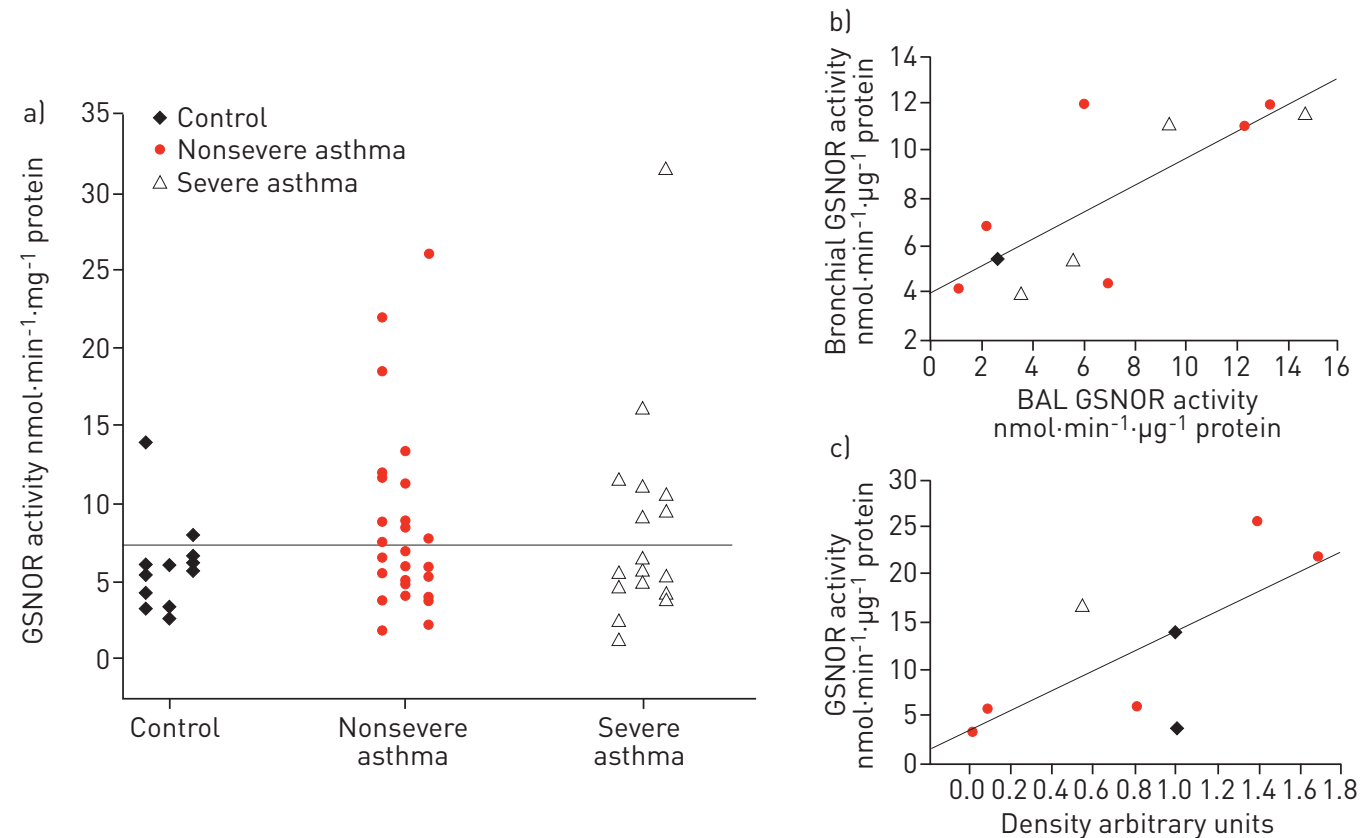

FIGURE 1 S-Nitrosoglutathione reductase (GSNOR) activity in asthma. a) GSNOR activity was measured in the bronchoalveolar lavage (BAL) cell lysate of 54 subjects, which included: control $n=13$, nonsevere asthma $n=25$ and severe asthma $n=16$. Severe and nonsevere asthma did not differ $(\mathrm{p}=\mathrm{Ns})$. A greater proportion of subjects with asthma had high activity $\left(>7.5 \mathrm{nmol} \cdot \mathrm{min}^{-1} \cdot \mu \mathrm{g}^{-1}\right.$ protein $)$ than did controls $(\mathrm{p}=0.032)$. b) A subset of subjects underwent analysis of an early (proximal) and late (distal) lavage fraction. Activities were weakly correlated between these two fractions $\left(\mathrm{r}^{2}=0.61\right)$. c) Consistent with the previous report, activity was roughly correlated with protein expression (measured by immunoblot, densitometry) $\left(\mathrm{r}^{2}=0.54\right)$. Activity was not correlated with BAL cell type ( $\left.\mathrm{p}=\mathrm{Ns}\right)$.

asthma native BAL cell pellets ex vivo, and in cultured epithelial cells in vitro, whereas IL-4 was not effective (fig. 3b, $c$ and d).

Consistent with previous reports [24, 27], HHe-3 imaging showed regions focal ventilatory defects in the lungs of subjects with severe asthma ( $\mathrm{n}=3$ ) (fig. 4). In figure $4 \mathrm{a}$ (coronal image) and figure $4 \mathrm{~b}$ (transverse image) ventilation defects are shown at baseline in stable, severe asthma patient. In figure $4 \mathrm{c}$ a second image acquired after albuterol administration shows only moderate improvement in the left upper lobe ventilation defect. Image-guided endobronchial biopsy done in the segmental airways supporting well-ventilated lung region (green arrows, fig. 4a) showed relatively preserved epithelial integrity, less GSNOR immunoreactivity and reduced inflammation (fig. $4 \mathrm{~g}-\mathrm{i}$ ) than biopsy taken from airways supporting region with focal ventilatory defects (red arrows, fig. $4 \mathrm{a}$ and $\mathrm{d}-\mathrm{f}$ ). Overall, when epithelium was present, it was immunostained more for GSNOR (3-4/4 in obstructed airways versus 2-3/4 in nonobstructed airways).

\section{Phenotype analysis of patients with increased GSNO reductase activity}

We next analysed the clinical phenotype of the well characterised SARP patients with evidence for increased airway GSNO reductase activity. Of the asthmatic subjects with BAL GSNO reductase activity measures, 31 were fully characterised in the SARP database (table 2). Those with GSNO reductase activity $>7.5 \mathrm{nmol} \cdot \mathrm{min}^{-1} \cdot \mu^{-1}$ protein had higher IgE $(\mathrm{p}=0.01)$ and were diagnosed with asthma and allergies at a younger age $(\mathrm{p}=0.02)$. Also, they were younger, had a lower body mass index (BMI), had a lower daytime sleepiness snores on obstructive sleep apnoea (OSA) screening and were less likely to have exercise-induced symptoms (each $\mathrm{p}<0.05$ ). Asthmatic subjects with increased GSNO reductase activity did not have a significantly lower methacholine $\mathrm{PC}_{20}$ (provocative concentration causing a $20 \%$ fall in forced expiratory volume in $1 \mathrm{~s}(\mathrm{FEV} 1)) \pm \mathrm{SD}(1.02 \pm 0.92)$ than those with low GSNO reductase activity $(4.32 \pm 6.79$, $\mathrm{p}=0.22$ ).

\section{GWAS data from the SARP}

One well-studied SNP in the GSNO reductase promoter associated with good responsiveness to $\beta_{2}$ adrenergic treatment (rs1154400) [15] was available on the Human1M-Duo DNA BeadChip used in the 

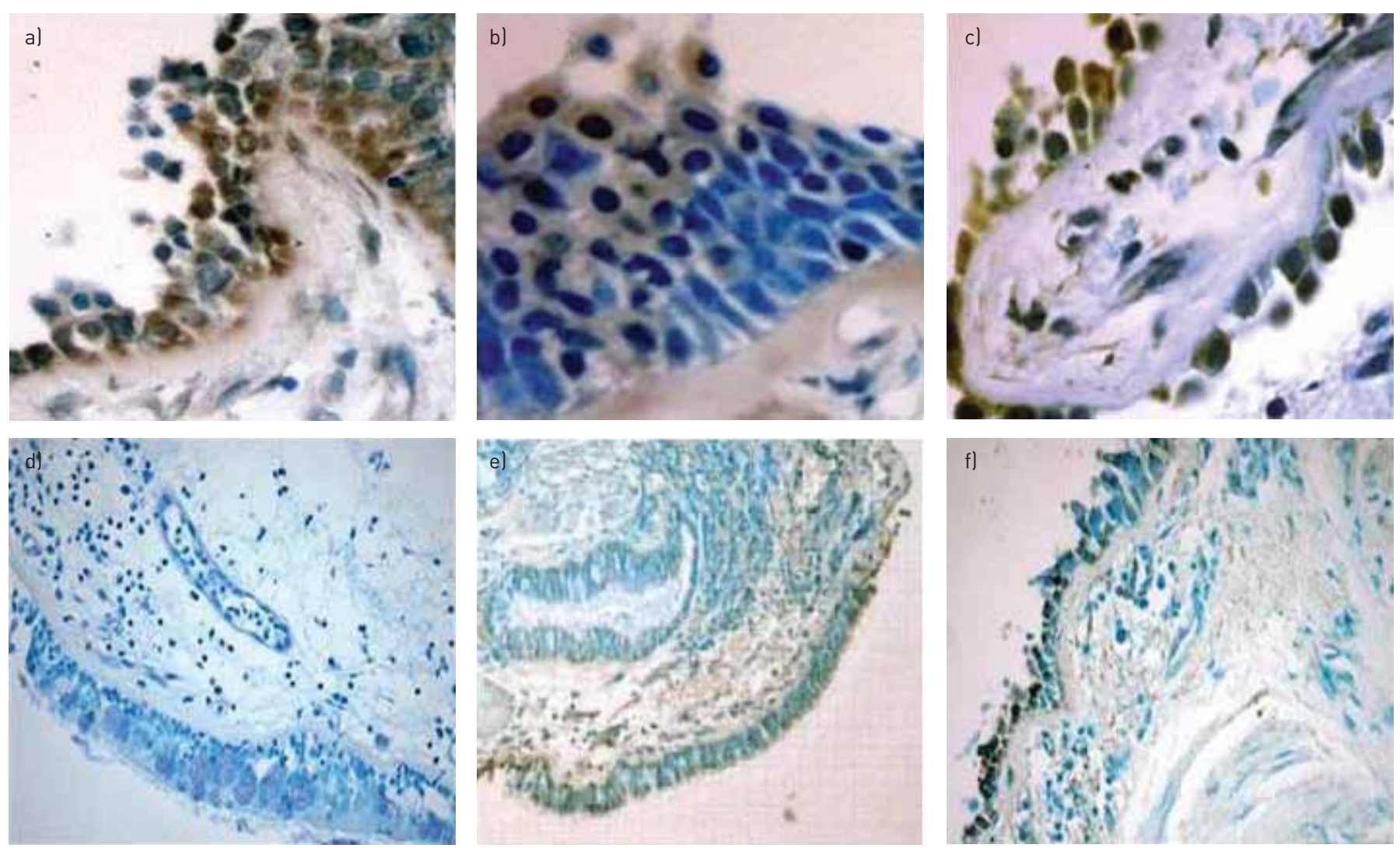

FIGURE 2 S-Nitrosoglutathione reductase (GSNOR) expression is primarily epithelial cells. Lower lobe biopsy sections were embedded in paraffin and immunostained for GSNOR as described in the methods section. a) Normal, control, lung showing GSNOR immunoreactivity in the pseudostratified airway epithelium. b) and e) Nonsevere asthma showing a similar distribution of GSNOR activity, particularly in the apex of the pseudostratified epithelium, less in basal membrane and smooth muscles. c) and f) Severe asthma showing loss of epithelium and thickening of the basal membrane; note the more prominent

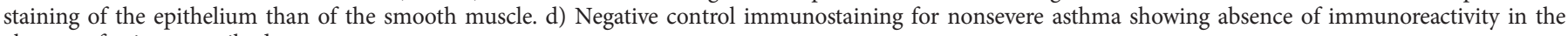
absence of primary antibody.

SARP. This SNP is in a region associated with protection from asthma in Caucasian populations [15-17] and the $\mathrm{C} / \mathrm{C}$ genotype was associated with improved asthma outcomes and $\beta_{2}$ responsiveness in an African-American population [15]; however, the authors did not measure airway GSNOR activity. The rs1154400 genotypes were in the Hardy Weinberg equilibrium in the SARP population. Of the subjects who had both BAL GSNO reductase analysis and GWAS, none with high GSNO reductase activity in BAL had the $\mathrm{C} / \mathrm{C}$ genotype, but the differences did not achieve significance because of sample size. In the SARP population as a whole, those asthmatics with $\mathrm{C} / \mathrm{T}$ and $\mathrm{T} / \mathrm{T}$ genotypes were more allergic than those with the $\mathrm{C} / \mathrm{C}$ genotype (IgE $362 \pm 846 \mathrm{IU} \cdot \mathrm{mL}^{-1}$ versus $245 \pm 374 \mathrm{IU} \cdot \mathrm{mL}^{-1}, \mathrm{n}=838$ and $\mathrm{n}=76$, respectively, $\mathrm{p}=0.028$ ); and, of those with a positive skin-prick test, wheal diameters were greater to Dermatophagoyetes farinae $(10.8 \pm 6.0 \mathrm{~mm}(\mathrm{n}=449)$ versus $8.8 \pm 4.5 \mathrm{~mm}(\mathrm{n}=42)$, respectively, $\mathrm{p}=0.009)$. Note that the $\mathrm{C} / \mathrm{T}$ and $\mathrm{C} / \mathrm{T}$ genotype patients had lower exhaled breath condensate $(\mathrm{EBC}) \mathrm{pH}$ than $\mathrm{C} / \mathrm{C}(7.7 \pm 0.8(\mathrm{n}=449)$ versus $8.0 \pm 0.5$ ( $\mathrm{n}=45)$, respectively, $\mathrm{p}=0.0004)$, consistent with data that high EBC formic acid levels may be associated with increased airway GSNO reductase activity [31]. Consistent with the data by Moore et al. [25], though $\mathrm{C} / \mathrm{C}$ did not differ from $\mathrm{C} / \mathrm{T}-\mathrm{T} / \mathrm{T}$ with regard to baseline $\mathrm{FEV} 1$ ( $\mathrm{p}=\mathrm{NS}$ ), FEV1 after four and six puffs of albuterol was higher in the $\mathrm{C} / \mathrm{C}$ group than in the $\mathrm{C} / \mathrm{T}-\mathrm{T} / \mathrm{T}$ group (four puffs $91.7 \pm 17.3 \%$ pred $(\mathrm{n}=81)$ versus $86.0 \pm 20.0 \%$ pred $(\mathrm{n}=697)$, respectively, $\mathrm{p}=0.013$; and six puffs $92.7 \pm 17.1 \%$ pred $(\mathrm{n}=72)$ versus $87.4 \pm 20.2 \%$ pred $(\mathrm{n}=697)$, respectively, $\mathrm{p}=0.034)$. The genotype groups did not differ in terms of age, sex or BMI.

\section{Discussion}

GSNO and other $S$-nitrosothiols cause airway smooth muscle relaxation in humans and other species [2, $4,32-34]$. Levels of S-nitrosothiols are low, and activity of GSNO reductase is high, in the airways of children with severe asthma who are experiencing an acute exacerbation associated with respiratory failure [13]. Here, we studied airway GSNO reductase expression and activity in stable outpatients with severe and non-severe asthma. 

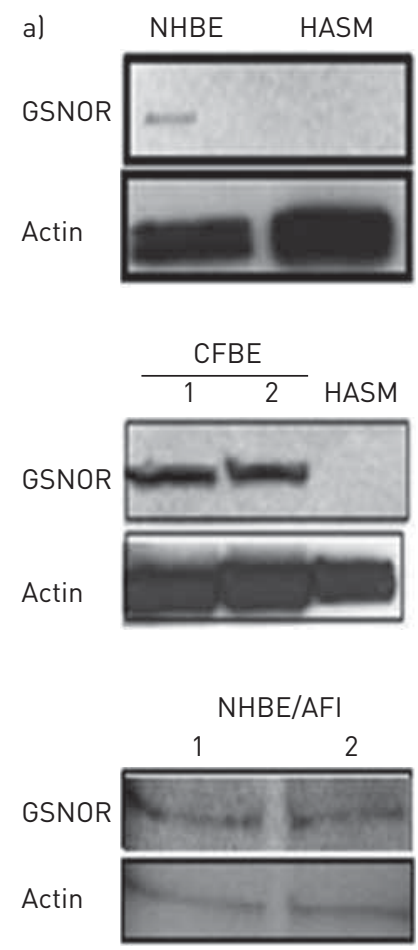
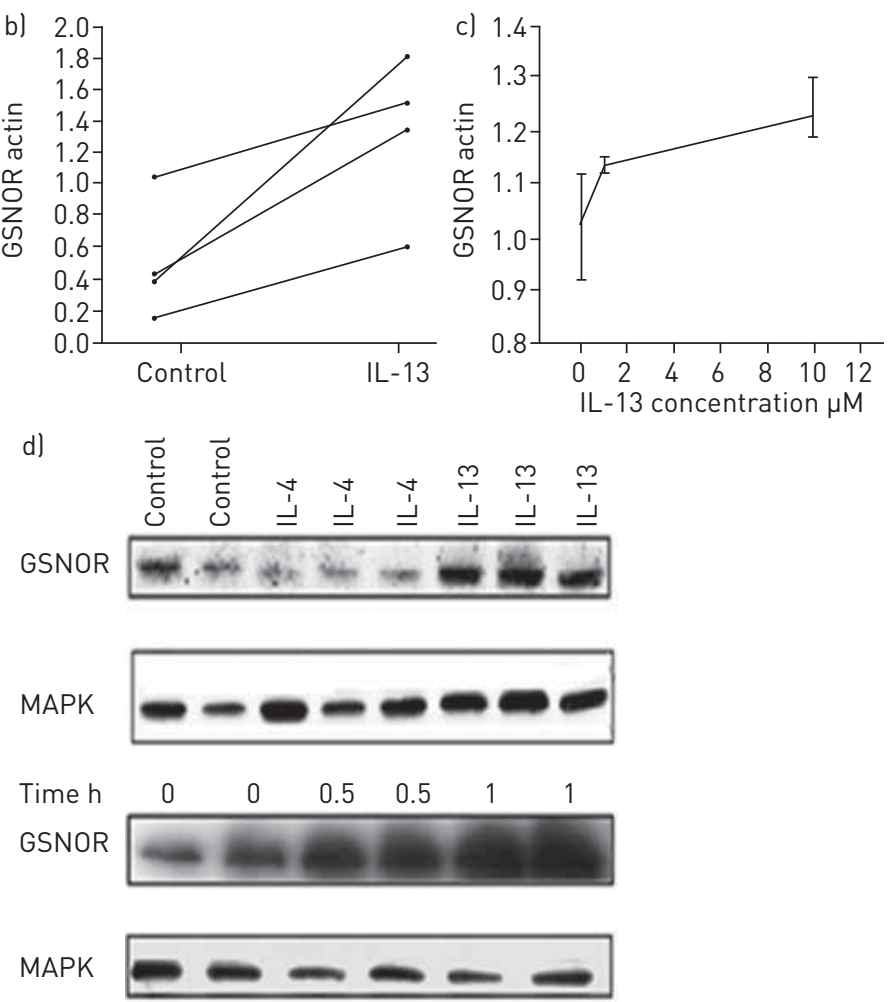

FIGURE 3 S-Nitrosoglutathione reductase (GSNOR) expression is greater in human airway epithelial cells than smooth muscle cells and is increased by interleukin (IL) 13. a) Immunoblots were performed on human normal bronchial epithelial cells (NHBE), both in monolayer culture and cultured at air-fluid interface (AFI). Studies were also performed on established cultures of human cystic fibrosis bronchial epithelial cells (CFBE) and on primary human airway smooth muscle (HASM) cells from six patients with asthma. Expression was greater in epithelial cells than in HASM cells, though there was variability in HASM expression. b) Freshly spun bronchoalveolar lavage cell pellets from severe asthma patients were exposed to IL-13 $\left(10 \mathrm{ng} \cdot \mathrm{mL}^{-1}\right.$ for $\left.4 \mathrm{~h}\right)$ ex vivo GSNOR expression was increased by IL-13 $(\mathrm{n}=4, \mathrm{p}<0.05)$. c) IL-13 incubation ( $2 \mathrm{~h}$ ) also increased GSNOR expression in A549 cells in a dose-dependent manner. d) IL-13, but not IL-4, increased GSNOR (10 ng. $\mathrm{mL}^{-1}$ each, $4 \mathrm{~h}$; $\mathrm{p}=0.05$ by ANOVA) relative to load control, and IL-13 (10 ng.mL $\left.\mathrm{mL}^{-1}\right)$ also caused a time-dependent, two-fold increase in GSNOR expression after $2 \mathrm{~h}$. MAPK: mitogen-activated protein kinase.

Activity of GSNO reductase was increased in the BAL cells of some stable outpatients with asthma. However, it was normal in many severe asthma patients; increased activity is not a defining pathophysiological feature of severe asthma. Consistent with the work of QuE et al. [10], activity was also normal in the BAL of a large proportion of stable, nonsevere asthmatics. Our data extends the observation to cases of severe asthma. In part, this is likely because there is population heterogeneity in GSNO reductase expression that is genetically determined. Previous studies have shown that increased airway GSNO reductase activity, and associated SNPs, are present only in a minority of patients with asthma [10, 14-16].

GSNO reductase has been of growing interest in the targeted asthma treatment [23]. It has been shown that that sputum glutathione disulfide levels were significantly decreased in patients with asthma, especially in the two inflammatory phenotypes. However, no correlation with the percentage of eosinophils or neutrophils was observed [12].

There is interest in treating asthma patients with GSNO reductase inhibition. Because a substantial chronic excess of $S$-nitrosothiols in the context of nitrosative stress has the potential to be associated with adverse effects $[5,18-20]$, it is important to target nitrosothiol repletion only to those patients who have an airway $S$-nitrosothiol deficiency and/or excessive GSNO catabolism associated with asthma. The SARP data suggest a specific phenotype. Patients with increased expression were more allergic, with earlier onset of asthma and allergies. They were younger and smaller, with reduced OSA. However, they were not more "severe" [22]. This proposed phenotype will require validation.

In a mouse model, GSNO inhalation modestly suppresses both T-helper cell type (Th)1 and Th2 cytokine responses, including IL-13 production, to antigen [5]. We also found that SARP subjects with a GSNO reductase-associated SNP, and associated with impaired $\beta_{2}$ responsiveness in Moore et al. [15] study, were more allergic. As predicted by Whalen et al. [3], data from CHOUdhry et al. [16] show that asthma and 

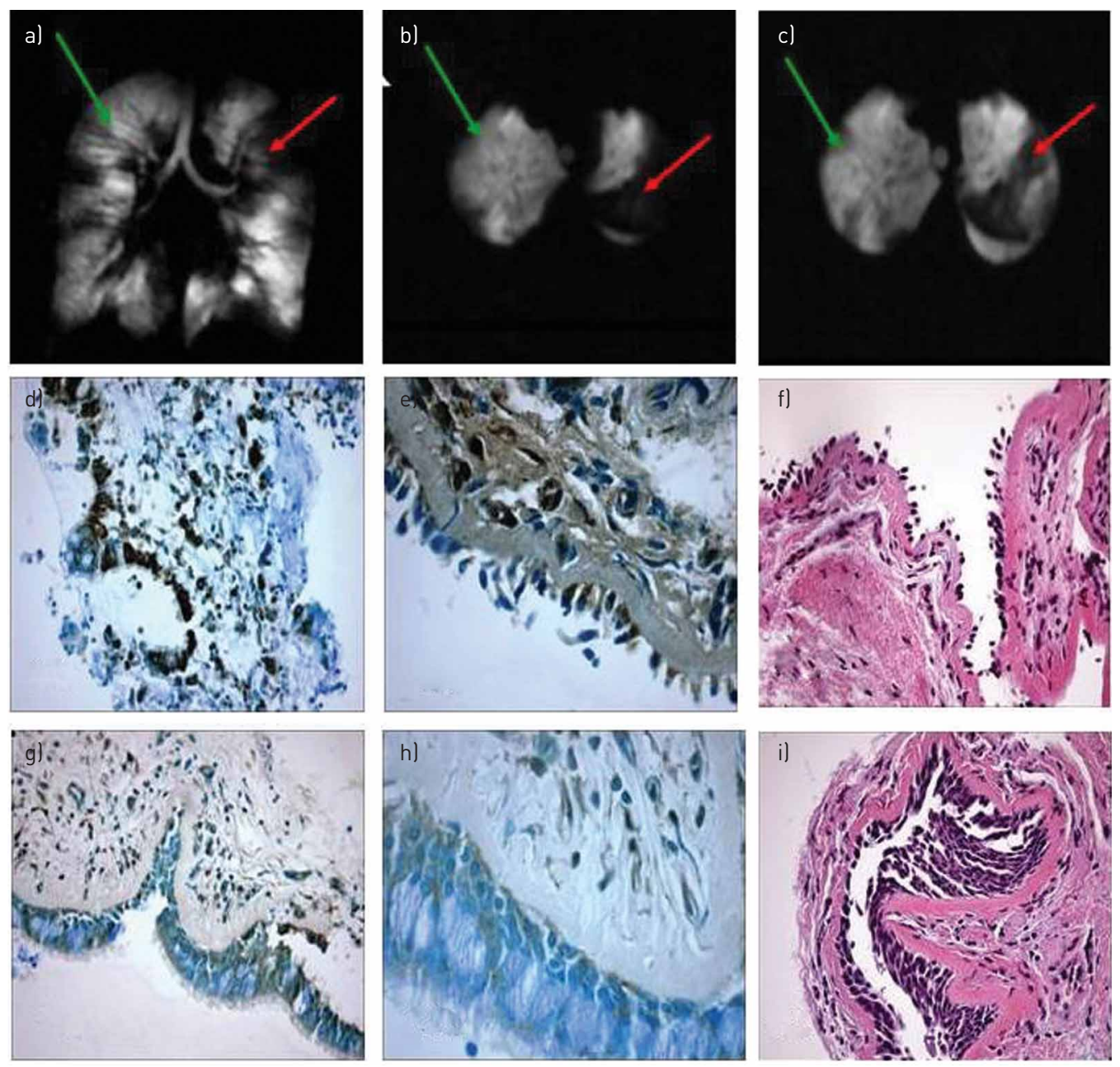

FIGURE 4 Regional inhomogeneity of inflammation and S-nitrosoglutathione reductase (GSNOR) expression in a severe asthma patient. On the day of the hyperpolarised helium magnetic resonance imaging scanning [24, 27], the radiologist reported to the bronchoscopist the regions of good ventilation and poor ventilation to guide the biopsies. Green arrows point to areas of good ventilation, and red arrows point to areas of poor ventilation. a) Coronal image and b) transverse image of the ventilation defects at baseline in this stable, severe asthma patient. c) A second image acquired after the administration of albuterol shows only moderate improvement in the left, upper-lobe ventilation defect. d, e) Inflammation and epithelial injury with increased GSNOR expression, in the poorly ventilated lung unit. f) Haematoxylin eosin staining, in a sample from the poorly ventilated lung unit, shows inflammation. g, h) GSNOR immunoreactivity in a sample from the well ventilated lung unit. i) Preserved airway epithelium, though thickened basement membrane, in a sample from the well ventilated lung unit.

impaired $\beta_{2}$ responsiveness are associated with genotypes causing GSNO reductase gain-of-function. Here, we have confirmed the finding that the bronchodilator responsiveness of subjects with $\mathrm{C} / \mathrm{C}$ was great than that of subjects with CT/and T/T [14]. This process may feed forward in asthma. Increased Th2 activation and IL-13 production would further increase GSNO reductase expression and activity (current study data) [5], particularly favouring increased GSNO reductase expression in allergic patients (fig. 5). We have shown that it is IL-13, not IL-4 that increases GSNOR expression. One of the possible mechanisms is that IL-13 can stimulate endothelial nitric oxide synthase activity, even in inflammatory cells. This effect can involve a feed-forward mechanism involving transforming growth factor $\beta 1$. In turn, these effects may stimulate GSNOR in a counter-regulatory fashion. However, more detailed cellular analysis will be required to confirm this hypothesis.

Bronchoscopic results can be flawed in asthma patients. Here, we have shown that GSNO reductase activity levels in patients may have reflected the lung region sampled, not simply overall phenotype. In our preliminary studies, expression was not uniform throughout the airway. One possible explanation is that the regional variations in expression result from treatment: inhaled corticosteroids may access the well-ventilated units, inhibiting inflammation and GSNO reductase expression. A previous HHe-3 study has shown that roughly $50 \%$ of poorly ventilated lung units have persistent defects [24], perhaps reflecting 
TABLE 2 Relationship between S-nitrosoglutathione reductase activity and risk factors: univariate analysis of subjects with complete datasets

\begin{tabular}{|c|c|c|c|}
\hline & $<7.5^{\#}$ & $>7.5^{\pi}$ & p-value \\
\hline $\mathrm{Age}^{+}$ & $38.65 \pm 10.5$ & $24.29 \pm 8.9$ & 0.001 \\
\hline Body mass index & $29.71 \pm 5.7$ & $24.09 \pm 7.0$ & 0.021 \\
\hline Female ${ }^{\S}$ & 17 & 8 & 0.174 \\
\hline Male & 2 & 4 & \\
\hline Severity subject classification $\%$ & & & 0.36 \\
\hline Not severe & $11 \pm 35.5$ & $9 \pm 29.0$ & \\
\hline Severe & $8 \pm 25.8$ & $3 \pm 9.7$ & \\
\hline Screening questionnaire: number of years with asthma & $23.95 \pm 10.80$ & $17.17 \pm 5.94$ & 0.032 \\
\hline Atopic diseases: age when allergies developed & $13.31 \pm 11.60^{f}$ & $5.18 \pm 4.84^{\# \#}$ & 0.020 \\
\hline Sleep and daytime alertness: sleep apnoea score & $29.19 \pm 7.25^{f}$ & $19.70 \pm 4.03^{\text {กาก }}$ & 0.001 \\
\hline Baseline pre-drug FEV $1 \mathrm{~L}$ & $2.40 \pm 0.75$ & $3.10 \pm 1.12$ & 0.044 \\
\hline Predicted FEV 1 /FVC Hankinson & $0.83 \pm 0.03$ & $0.85 \pm 0.02$ & 0.007 \\
\hline Blood Log10 $\lg E$ & $1.78 \pm 0.64^{++}$ & $2.47 \pm 0.66^{\pi}$ & 0.008 \\
\hline $\begin{array}{l}\text { Asthma symptoms caused by routine physical activity, } \\
\text { provoking factors } \%\end{array}$ & $13 \pm 44.8$ & $2 \pm 6.9$ & 0.008 \\
\hline
\end{tabular}

Data are presented as mean $\pm S D$, unless otherwise stated. FEV 1 : forced expiratory volume in $1 \mathrm{~s}$; Hankinson: refers to the normal spirometry data. ${ }^{\#}: n=19 ;{ }^{\uparrow}: n=12 ;{ }^{+}$: age at enrolment into Severe Asthma Research Program; ${ }^{\S}$ : using one-sample proportions exact test, there was a greater proportion of females in the $<7.5$ category than in the $>7.5$ category $(p=0.001) ;{ }^{f}: n=16 ;{ }^{\# \#}: n=11 ;{ }^{\text {ๆा }}: n=10 ;{ }^{++}: n=18$.

chronic lack of therapy. It is also possible that some airways, like the skin, have inherently different patterns of inflammation. Either way, it is important to recognise that there may be substantial intra-subject airway heterogeneity. Ongoing studies in the SARP and other programmes are investigating this regional variability more prospectively.

Further, bronchoscopy is not without expense and potential risk. Therefore, noninvasive biomarkers may prove useful for diagnosing of the severe asthma. Possibly consistent with low $\mathrm{pH}$ in the non-C/C genotype population, our previous data suggest that increased EBC formic acid levels might be a noninvasive marker for increased GSNO reductase activity [31]. We have previously shown that inhaled GSNO increases exhaled nitric oxide, followed by time-dependent decay [35]. Therefore, an inhalational challenge with GSNO may test for the presence and/or the disinhibition of GSNO reductase activity in human asthmatics: the greater the activity of GSNO reductase, the higher the fractional exhaled nitric oxide (FeNO) after challenge. Airway homogeneity, in turn, could be assayed using different FeNO flow rates. We believe that this kind of challenge test, and/or EBC formic acid test, may prove clinically useful to identify severe asthma patients who could be candidates for $S$-nitrosothiol replacement and/or inhibition of GSNO reductase.

Of note that is that we found increased GSNO reductase activity in asthmatics was not significantly associated with methacholine PC20, though there was a trend in this direction. This could be explained by corticosteroid treatment. In our study $83 \%$ of patients with nonsevere and $100 \%$ of patients with severe asthma received inhaled steroids. In the study by QuE et al. [10], only subjects with mild asthma were included, and they did not receive either a high-dose inhaled or oral corticosteroids.

In summary, we found marked heterogeneity in GSNO reductase activity in subjects with both severe and nonsevere asthma. Increased GSNO reductase activity is not, in general, the cause of severe asthma. We also present evidence that patients with increased airway GSNO reductase activity may have a more

FIGURE 5 Proposed scheme showing the effect of increased $S$-nitrosoglutathione reductase (GSNOR) on asthma. A subset of patients, as previously reported $[10,14-16]$, has a diathesis to increased GSNOR expression. This is driven by allergic airway inflammation. In turn, this causes a decrease in airway GSNO levels that leads smooth muscle constriction $[1-7,32-34]$ and a further increase in T-helper cell (Th)2 cytokine expression [5].

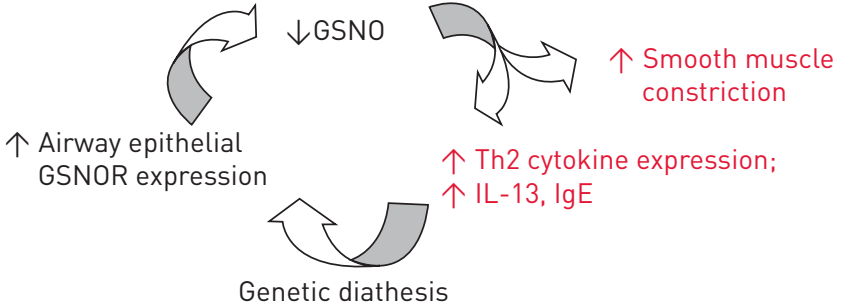

Genetic diathesis 
allergic phenotype. If validated, this high GSNO reductase phenotype could be suspected clinically based on history and physical examination and confirmed with genetic analysis and/or with biomarker testing in the lung function laboratory. This information, in turn, can be used to personalise treatment. We believe that this paradigm will be of increasing value in subspecialty asthma management.

\section{Acknowledgements}

We would like to thank all the Severe Asthma Research Program coordinators.

\section{References}

1 Gaston B, Reilly J, Drazen JM, et al. Endogenous nitrogen oxides and bronchodilator S-nitrosothiols in human airways. Proc Natl Acad Sci USA 1993; 90: 10957-10961.

2 Gaston B, Drazen JM, Jansen A, et al. Relaxation of human bronchial smooth muscle by $S$-nitrosothiols in vitro. J Pharmacol Exp Ther 1994; 268: 978-984.

3 Whalen EJ, Foster MW, Matsumoto A, et al. Regulation of $\beta$-adrenergic receptor signalling by $S$-nitrosylation of G-protein-coupled receptor kinase 2. Cell 2007; 129: 511-522.

4 Gaston B, Doctor A, Singel D, et al. S-nitrosothiol signaling in respiratory biology. Am J Respir Crit Care Med 2006; 173: 1186-1193.

5 Foster M, Yang Z, Potts E, et al. S-nitrosoglutathione supplementation to ovalbumin-sensitized and -challenged mice ameliorates methacholine-induced bronchoconstriction. Am J Phyisol Lung Cell Mol Physiol 2011; 301: 739-744.

6 Fang K, Johns R, Macdonald T, et al. S-nitrosoglutathione breakdown prevents airway smooth muscle relaxation in the guinea pig. Am J Physiol Lung Cell Molec Physiol 2000; 279: L716-L721.

7 Forster MW, Hess DT, Stamler JS. Protein S-nitrosylation in health and disease: a current perspective. Trends Mol Med 2009; 15: 391-404.

8 Liu L, Hausladen A, Zeng M, et al. A metabolic enzyme for S-nitrosothiol conserved from bacteria to humans. Nature 2001; 410: 490-494.

9 Que LG, Liu L, Yan Y, et al. Protection from experimental asthma by an endogenous bronchodilator. Science 2005; 308: 1618-1621.

10 Que L, Zhonghui Y, Stamler JS, et al. S-nitrosoglutathione reductase: an important regulator inhuman asthma. Am J Respir Crit Care Med 2009; 180: 226-231.

11 Xiong Y, Uys JD, Tew KD, et al. S-glutathionylation: from molecular mechanisms to health outcomes. Antioxid Redox Signal 2011; 15: 233-270.

12 Kuipers I, Louis R, Manise M, et al. Increased glutaredoxin-1 and decreased protein S-glutathionylation in sputum of asthmatics. Eur Respir J 2013; 41: 469-472.

13 Gaston B, Sears S, Woods J, et al. Bronchodilator S-nitrosothiol deficiency in asthmatic respiratory failure. Lancet 1998; 351: 1317-1319.

14 Wu H, Romieu I, Sienra-Monge JJ, et al. Genetic variation in S-nitrosoglutathione reductase (GSNOR) and childhood asthma. J Allergy Clin Immunol 2007; 120: 322-328.

15 Moore PE, Ryckman KK, Williams SM, et al. Genetic variants of GSNOR and ADRB2 influence response to albuterol in African-American children with severe asthma. Pediatr Pulmonol 2009; 44: 649-654.

16 Choudhry S, Que LG, Yang Z, et al. GSNO reductase and $\beta_{2}$-adrenergic receptor gene-gene interaction: bronchodilator responsiveness to albuterol. Pharmacogenet and Genomics 2010; 20: 351-358.

17 Green LS, Chun LE, Patton AK, et al. Mechanism of inhibition for N6022, a first-in-class drug targeting S-nitrosoglutathione reductase. Biochem 2012; 51: 2157-2168.

18 Palmer L, Doctor A, Chhabra P, et al. S-nitrosothiols signal hypoxia-mimetic vascular pathology. J Clin Invest 2007; 117: 2592-2601.

19 Marozkina NV, Wei C, Yemen S, et al. S-nitrosoglutathione reductase in human lung cancer. Am J Respir Cell Mol Biol 2012; 46: 63-70.

20 Wei W, Li B, Hanes MA, et al. S-nitrosylation from GSNOR deficiency impairs DNA repair and promotes hepatocarcinogenesis. Sci Transl Med 2010; 2: 19ra13

21 Moore WC, Bleecker ER, Curran-Everett DC, et al. Characterization of the severe asthma phenotype by the National Heart, Lung. and Blood Institutes's Severe Asthma Research Program. J Allergy Clin Immunol 2007; 119: 405-413.

22 Busse WW, Banks-Schlegel S, Wenzel SE. Pathophysiology of severe asthma. J Allergy Clin Immunol 2000; 106: 1033-1042.

23 Chung KF, Wenzel SE, Brozek JL, et al. International ERS/ATS guidelines on definition, evaluation and treatment of severe asthma. Eur Respir J 2014; 43: 343-373.

24 de Lange EE, Altes TA, Patrie JT, et al. Changes in regional airflow obstruction over time in the lungs of patients with asthma: evaluation with 3He MR imaging. Radiology 2009; 250: 567-575.

25 Moore WC, Evans MD, Bleecker ER, et al. Safety of investigative bronchoscopy in the Severe Asthma Research Program. J Allerg Clin Immunol 2011; 128: 328-336.

26 Fitzpatrick AM, Brown LA, Holguin F, et al. Levels of nitric oxide oxidation products are increased in the epithelial lining fluid of children with severe asthma. J Allergy Clin Immunol 2009; 124: 990-996.

27 Fain SB, Gonzalez-Fernandez G, Peterson ET, et al. Evaluation of structure-function relationships in asthma using multi-detector CT (MDCT) and hyperpolarized (HP) He-3 MRI. Acad Radiol 2008; 15: 753-762.

28 Gow A, Doctor A, Mannick J, et al. S-nitrosothiol measurements in biological systems. J Chromatog B Analyt Technol Biomed Life Sci 2007; 851: 140-151.

29 Amrani Y, Syed F, Huang C, et al. Expression and activation of the oxytocin receptor in airway smooth muscle cells: regulation by TNFo and IL-13. Respir Res 2010; 11: 104

30 Marozkina NV, Yemen S, Borowitz M, et al. Hsp70/Hsp 90 organizing protein as a nitrosylation target of corrector therapy in cystic fibrosis therapy. Proc Natl Acad Sci USA 2010; 107: 11393-11398. 
31 Greenwald R, Fitzpatrick AM, Gaston B, et al. Breath formate is a marker of airway S-nitrosothiol depletion in severe asthma. PLoS One 2010; 5: e11919

32 Bannenberg G, Xue J, Engman L, et al. Characterization of bronchodilator effects and fate of S-nitrosothiols in the isolated perfused and ventilated guinea pig lung. J Pharmacol Exp Ther 1995; 272: 1238-1245.

33 Janssen LJ, Premji $\mathrm{M}$, Lu-Chao $\mathrm{H}$, et al. $\mathrm{NO}^{+}$but not $\mathrm{NO}$ radical relaxes airway smooth muscle via cGMP-independent release of internal Ca2 ${ }^{+}$. Am J Physiol Lung Cell Mol Physiol 2000; 278: 899-905.

34 Perkins W, Pabelick C, Warner DO, et al. cGMP-independent mechanism of airway smooth muscle relaxation induced by S-nitrosoglutathione. Am J Physiol 1998; 275: 468-474.

35 Snyder A, McPherson ME, Hunt JF, et al. Acute effects of aerosolized S-nitrosoglutathione in cystic fibrosis. Am J Respir Crit Care Med 2002; 165: 922-926. 